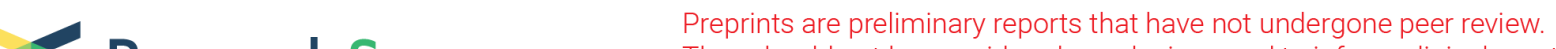 Research Square
or referenced by the media as validated information.
}

\section{MiR-224 Promotes Lymphatic Metastasis by Targeting ANGPTL1 in Non-Small-Cell Lung Carcinoma}

Haibo Han

Peking University Cancer Hospital \& Institute Beijing

Bo Pan

Peking University Cancer Hospital \& Institute Beijing

Fan Liang

Peking University Cancer Hospital \& Institute Beijing

Lina Wu

Peking University Cancer Hospital \& Institute Beijing

Xijuan Liu

Peking University Cancer Hospital \& Institute Beijing

Yue Yang

Peking University Cancer Hospital \& Institute Beijing

Jin-feng Chen ( $\nabla$ chenjinfengdoctor@163.com )

Peking University Cancer Hospital \& Institute Beijing

\section{Research Article}

Keywords: microRNA, miR-224, non-small-cell lung carcinoma, lymphatic metastasis

Posted Date: June 1st, 2021

DOl: https://doi.org/10.21203/rs.3.rs-464176/v1

License: (c) (i) This work is licensed under a Creative Commons Attribution 4.0 International License.

Read Full License

Version of Record: A version of this preprint was published at Cancer Biomarkers on March 3rd, 2022. See the published version at https://doi.org/10.3233/CBM-210376. 
MiR-224 promotes lymphatic metastasis by targeting ANGPTL1 in non-small-cell lung carcinoma

Haibo Han ${ }^{1}$, Bo $\mathrm{Pan}^{2}$, Fan Liang ${ }^{2}$, Lina $\mathrm{Wu}^{3}$, Xijuan $\mathrm{Liu}^{3}$, Yue Yang${ }^{2}$, and Jinfeng Chen $^{2 *}$

${ }^{1}$ Department of Clinical Laboratory, ${ }^{2}$ Department of Thoracic Surgery II, ${ }^{3}$ Department of Central Laboratory, Key laboratory of Carcinogenesis and Translational Research (Ministry of Education/Beijing), Peking University Cancer Hospital \& Institute Beijing, China;

Correspondence to: Jin-feng Chen Ph.D., M.D, Key Laboratory of Carcinogenesis and Translational Research (Ministry of Education), Department of Thoracic Surgery II, Peking University Cancer Hospital \& Institute, 52 Fucheng Road, Haidian District, Beijing 100142, Email: chenjinfengdoctor@163.com 


\section{Abstract}

Background: MicroRNAs can regulates tumor metastasis either as an oncomiR or suppressor miRNA. Here, we investigate the role of miR-224 in lymphatic metastasis of non-small-cell lung cancer (NSCLC).

Methods: The expression of miR-224 was demonstrated by a validation cohort of 156 lung cancer patients (77 cases with lymphatic metastasis) by q-PCR. In vitro and in vivo experiments were performed to study the malignant phenotype after upregulation and inhibition of miR-224 expression. Furthermore, the direct target genes of miR-224 were determined by a luciferase reporter assay.

Results: miR-224 was identified as a high expression miRNA in the tumor tissues with lymphatic metastasis) with an area under the receiver operating characteristic curve (AUC) of 0.57. Forced expression of miR-224 in H1299 cells promoted not only the cell viability, plate clone formation, migration and invasion in vitro, but also tumor growth and lung metastasis in vivo. Consistently, inhibition of miR-224 suppressed the malignant characters both in vitro and in vivo. Molecular mechanism research suggested that miR-422a targeted the ANGPTL1 as a novel tumor suppressor.

Conclusions: The present study demonstrates that miR-224 is a potential marker for the prediction of lymphatic metastasis of NSCLC. And application of miR-224 may help for prophylactic intervention of NSCLC in clinical practice.

Keywords: microRNA, miR-224, non-small-cell lung carcinoma, lymphatic metastasis 


\section{Background}

Globally, lung cancer is the leading cause of cancer death [1] and its 5-year survival rate is about $4-17 \%[2]$. Among two pathological subtypes, non-small cell lung cancer (NSCLC) is the most common type, accounting for about $80 \%$ of lung cancer [3]. Lymph node metastasis (LNM) was an independent factor affecting survival and recurrence after surgical resection of the primary malignancy [4]. Patients with mediastinal lymph node dissection was associated with lower local recurrence rates and better survival [5].Therefore, searching for LNM-related MicroRNAs (miRNAs or miRs) and understanding the molecular mechanisms was deeply meaningful.

MiRNAs were defined as endogenous and small RNAs of $\sim 22 \mathrm{nt}$ nucleotides in length by complementary base pairing with the target mRNA, leading to its degradation [6]. Recent discoveries related to miRNA alterations in cancer, either as oncomiR or suppressor miR. Thus modulating miRNA expression and activity in vivo through miRNA mimics or antimiRs provides an opportunity for the development of innovative therapeutic approaches to cancer [7]. Previous studies demonstrated that numerous miRNAs correlated with LNM of NSCLC [8]. In recent years many miRNAs have been identified to be associated with LNM, including miR-137 [9], miR-144-3p [10]as metastasis inducer, and miR-148a [11], miR-129-5p [12] as metastasis suppressor.

Previously, we had identified the differentially expressed miRNAs in patients with lymph node metastasis (LNM) compared with the patients without lymph node metastasis by miRNAs microarray analysis, and the up-regulated expression of miR224 was identified as a high risk for LNM..In this study, we selected miR-224, as a potential LNM promotor, to explore its role on metastasis by both in vitro and in vivo experiments.

\section{Methods}




\section{Cell lines and culture conditions}

Human lung cancer cell lines (H1299 and PC9) were purchased from Chinese

Academy of Medical Sciences \& Peking Union Medical College (Beijing, China).

Cells were cultivated in RPMI 1640 with $10 \%$ FBS, $100 \mathrm{U} / \mathrm{mL}$ penicillin, and 100

$\mathrm{mg} / \mathrm{mL}$ streptomycin in a $5 \% \mathrm{CO} 2$ humidified incubator at $37^{\circ} \mathrm{C}$.

\section{Patients and samples}

A cohort of 155 NSCLC patients (77 cases with lymphatic metastasis and 78 patients without lymphatic metastasis) was included from 2003 to 2019 to validate miR-224 expression in cancer tissues. Inclusion criteria: 1)definitive pathologic diagnosis; 2) no chemoradiotherapies before surgery; 3) who completed a 5-year follow-up; 4) with written form informed consent. Exclusion criteria: 1) other types of clinical disorders were observed; 2) patients lost during follow-up or died of other clinical disorders. This study was approved by the Ethics Committee of Peking University.

\section{Quantitative real-time polymerase chain reaction (qRT-PCR)}

MiRNAs were extracted from tissues samples from NSCLC patients or cells using miRNeasy Mini Kit (Qiagen, Valencia, CA, USA) according to the manufacturer's instructions. The purity and concentration of RNA was estimated using the ND-1000 microspectrophotometer (Thermo Fisher Scientific, Waltham, MA, USA). For mature miRNA expression detection, the polyA tailing method was used [13], and cDNA were synthesized from $100 \mathrm{ng}$ RNA using the moloney murine leukaemia virus reverse transcriptase (M-MLV RT) (Invitrogen, Carlsbad, CA). For mRNA expression detection, $2 \mu \mathrm{g}$ of total RNA were used to synthesize cDNA. Quantitative real-time PCR (qRT-PCR) was performed using SYBR Green PCR Master Mix (ABI) at $95^{\circ} \mathrm{C}$ for 10 min followed by 40 cycles of $95^{\circ} \mathrm{C}$ for $15 \mathrm{~s}$ and $60^{\circ} \mathrm{C}$ for $60 \mathrm{~s}$ on $\mathrm{ABI} 7500$ System (Applied Biosystems, CA). The expression level of gene was calculated by the $2^{-\Delta \mathrm{Ct}}$ 
method, where $\Delta \mathrm{Ct}=\mathrm{Ct}$ (target gene)-Ct (GAPDH or U6). The sequences of all primers were on the list of supplementary Table S1.

\section{Cell viability assay}

$5 \times 10^{3}$ cells/well were seeded into 96-well plates and cultured for 24, 48, 72, and $96 \mathrm{~h}$. Cell Counting Kit-8 (CCK8; Dojindo, Kumamoto, Japan) reagents were added to cultures and incubated for additional one hour. The absorbance at $450 \mathrm{~nm}$ was measured by a microplate reader (iMark, Bio-Rad Laboratories, Hercules, CA).

\section{Plate colony formation assay}

500 cells/well were plated in 6 -well plates and cultured for 2 weeks. The number of cell colonies was counted after fixed with $4 \%$ formalin and stained with $1 \%$ crystal violet.

\section{Transwell assay}

For cell migration and invasion (coated with extracellular matrix substitute) assay, mitomycin C-treated cells were loaded into the upper chamber of Transwell insert with $8.0 \mu \mathrm{m}$ polycarbonate membranes (Millipore, Billerica, MA, USA) containing 1\% FBS in RPMI-1640 medium. Medium containing 10\% FBS was added into the lower chamber as a chemoattractant. Cells on the lower surface were stained with $1 \%$ crystal violet and photographed under a microscope for four random fields per well.

\section{Wound healing assay}

Cells were cultured in $24-w e l l$ plates and scratched with a $20 \mu \mathrm{L}$ pipette tip. Detached cells were removed carefully with PBS wash for three times and the wounded area was photographed at 0, and $24 \mathrm{~h}$ (Leica, Wetzlar,Germany).

\section{In vivo tumor growth and metastasis assay}

Animal experiments were approved by Animal Ethics Committee of Peking University Cancer Hospital \& Institute. The in vivo tumor growth and metastatic characteristics of the cells were measured by a modified chick embryo chorioallantoic 
membrane assay $(\mathrm{CAM})$. Briefly, $5 \times 10^{6}$ cells pre-stained by the cell tracker dye CMDil (red fluorescence) were loaded on the CAM with 10- day-old chick embryos. Seven days later, the tumors on the CAM were dissected and weighed. The lungs of chick embryos were isolated and the metastatic tumor lesions with red fluorescence were evaluated under a fluorescence microscope (Leica, Germany).

\section{Analysis of microRNAs predicted targets by online software}

The predicted targets of the microRNAs were analyzed by an online database, miRecords (http://c1.accurascience.com/miRecords/), which is a resource for animal miRNA-target interactions. The Predicted Targets component of mIRecords integrates the predicted targets of the following miRNA target prediction tools: DIANA-microT, MicroInspector, miRanda, MirTarget2, miTarget, NBmiRTar, PicTar, PITA, RNA22, RNAhybrid, and TargetScan/TargertScanS [14].

\section{Plasmid construction}

The ANGPTL1 and YPEL1 3'-UTR fragments containing the miR-224 complementary binding sites and corresponding mutants were inserted into the pGL3.0-control (Promega, Madison, WI) downstream of the luciferase coding sequence, and confirmed by sequencing.

\section{Dual-luciferase reporter assays}

HEK-293FT cells cultured in 24-well plates were transfected with pGL-3 construct (300 ng), miR-224 mimic or negative control (NC: $50 \mathrm{nM}$ ) and Renilla luciferase construct (20 ng) using Lipofectamine 2000 (Invitrogen). Firefly and Renilla luciferase activities were dected by a dual luciferase assay (Promega) $24 \mathrm{~h}$ after transfection, and firefly luciferase activity normalized to Renilla luciferase activity was caluculated.

\section{Western blotting analysis}


Cells were lysised with RIPA lysis buffer with protease inhibitors and the protein was quantified by BCA method. Equal amounts $(30 \mu \mathrm{g})$ of protein were separated by $10 \%$ SDS-PAGE and transferred onto PVDF membranes (Millipore, Bedford, MA). The membranes were incubated with primary antibodies, followed by HRP-linked secondary antibodies. Signals were detected using an enhanced chemiluminescence assay (Thermo Scientific, Rockford, IL).

\section{Statistical analysis}

Statistical analyses were calculated with SPSS 16.0 (IBM, Armonk, NY, USA). Receiver operating characteristic (ROC) curves were established for discriminating metastatic lymph node and noncancerous lymph node. Two-tailed chi-squared test $\left(\chi^{2}\right)$ was used to evaluate the relationship between miR-224 expression and clinicopathological factors. Survival was analyzed by the Kaplan-Meier method using log-rank test. Continuous variable with normal distribution are expressed as means \pm SD. Two-sided Student's t-test, unless specified was used for difference comparison between two groups. ANOVA analysis followed by Bonferroni's post hoc test test were used to analyze differences among multiple groups. A P-value of $<0.05$ was considered significant.

\section{Results}

\section{Validate the miR-224 expression in NSCLC with lymphatic metastasis}

Previously, 50 miRNAs were identified to be differentially expressed by miRNA microarray analysis in lymph node tissues with metastasis from five NSCLC patients and compared with that in the corresponding noncancerous lymph node tissue, [15]. Among these miRNAs, miR-224 was significantly up-regulated in metastatic lymph nodes with 14.6-time fold change (Fig. 1A). Then, we validated miR-224 in a cohort of 155 cases of patients. Consistently, the miR-224 expression in samples with lymphatic 
metastasis was significantly higher than those without lymphatic metastasis (about 6.8 -time fold change, Fig. 1B, $P=0.0005)$, and showed an AUC value of 0.744 compared in evaluating lymphatic metastasis (Fig. 1C). Moreover, a survival prognostic analysis by the Kaplan-Meier method revealed that high expression of miR-224 in NSCLC tissues was associated with poor overall survival (Fig. 1D, $P=0.001$ ).

\section{Forced expression of miR-224 enhanced cell viability, colony formation, migration} and invasion in $\mathbf{H 1 2 9 9}$ cells in vitro

Firstly, the expression of miR-224 was detected by qRT-PCR in six lung cancer cell lines. As shown in Fig. 2A. The miR-224 expression was relative high in the PC9, A549 and H520 cells, but low in the GLC82, H1299 and H1975 cells. Therefore, we used the H1299 cells for over-expression experiment and PC9 for knockdown experiment to reveal the role of miR-224 in malignant phenotype for lung cancer cells. As shown in Fig. 2B, miR-224 expression was increased by about 40 -fold after transfected with mimic at 24 hrs. Forced expression of miR-224 remarkably promoted cell viability (Fig.2C, $P<0.0001$ ) and colony formation ability by two times (Fig.2D, $P<0.0001)$. Furthermore, following the increased miR-224, the spreading of H1299 cells was much faster than that of the NC cells by wound healing assay (Fig.2E, $P<$ 0.0001). Compared with NC cells, the migration and invasion ability of H1299 cellls transfected with miR-224 mimics were also remarkably increased as determined by transwell assay (Fig.2F, $P<0.001)$.

\section{Inhibition of miR-224 suppresses PC9 cell migration and invasion in vitro}

MiR-224 inhibitor was transfected into PC9 cells with relative high endogenous expression of miR-224. MiR-130a-3p expression was successfully decreased by $71 \%$ 
1 after cells transfected with inhibitor (Fig. 3A). Consistent with the results above, inhibition of miR-1224 decreased cell viability (Fig. 3B, $P<0.01$ ) and colony formation ability (Fig. 3C, $P<0.01$ ). Moreover, inhibition of miR-224 remarkably inhibited wound closure in wound healing assay (Fig. 3D, $P<0.001$ ). The cell number of migration and invasion through Matrigel were also decreased in transwell assays (Fig. 3E, $P<0.001)$.

\section{MiR-224 functions as oncomiR in vivo}

To validate the result above, we employed a modified chick embryo chorioallantoic membrane (CAM) assay to assess the role of miR-224 on tumor growth and metastasis in vivo. As shown in Fig. 4A, over-expression of miR-224 in H1299 cells resulted in 5.2-fold-time increase in tumor weight compared to control cells (tumor weight, $P=0.03$ ). Moreover, metastastic lung lesions were also increased by 2.8 -fold-time in miR-224-over-expressing cells (Fig. 4B, $P=0.03$ ). Consistently, compared with control cells, the tumor weights formed from the miR-224 inhibited PC9 cells was decreased by $83.8 \%$ on CAM assay(Fig. $4 \mathrm{C}$, tumor weight, $P=0.02$ ). Furthermore, the metastatic cancer cells to embryo lungs were also markedly decreased by $83.0 \%$ in miR-224inhibited group (Fig. 4D, $P=0.02$ ). Collectively, these data indicated that MiR-224 functions as oncomiR both in vitro and in vivo, which can enhance malignant phenotype of NSCLC.

\section{ANGPTL1 was a direct target of MiR-224 involved in its oncogene function}

As we all knew, the biological significance of miRNAs relied on its regulation on their targets. Firstly, we analyzed the miRNA targets by the online database, miRecords. 
Given the hypothesis that miR-224 correlated with lymphatic metastasis, we analyzed the differential expressed genes (DEGs) of lung cancer tissues at N1-3 stage with lymph node metastasis compared with those at N0 stage without lymph node metastasis, and we performed correlation analysis between the mRNA and the miRNA expression profile from the Cancer Genome Atlas (TCGA) database. A three-set Venn diagram showed 41 intersections of candidate targets (Fig. 5A). Of the 41 predicted target genes, we selected two tumor suppressor genes ANGPTL1 and YPEL1 for further validation. We detected the expression levels of ANGPTL1 and YPEL1 in miR-224 overexpression and inhibition cells. As shown in Fig. 5B, the expression of two candidate genes were both remarkably decreased in miR-224 over-expression H1299 cells, whereas increased in PC9 cells transfected with miR-224 inhibitor at protein level. Both ANGPTL1 and YPEL1 had one consensus binding sites in their 3'-UTR. Luciferase reporters containing either the wild type or mutant type 3'-UTR fragment were constructed, respectively (Fig. 5C). Dual-luciferase reporter assays showed that miR-224 mimic significantly reduced the reporter activity of wild type of ANGPTL1 luciferase construct (Fig. 5D, $P<0.01$ ), but not the corresponding mutant construct (Fig. 5D, $P>0.05)$. However, there was no significant deference for the luciferase activity between miR-224 mimic and NC groups both for wild and mutant type of YPEL1 luciferase construct (Fig. 5D, $P>0.05$ ). These data suggested that miR-224 directly regulates ANGPTL1 expression by binding to the 3'-UTR region of its mRNA, but indirectly inhibited YPEL1 expression. We performed dual luciferase reporter assays. Moreover, the data download from TCGA (Fig. 5E left graph, Spearman r = - 0.1536, $P=0.0009)$ and from our cohort of 155 cases of lung cancer patients all displayed a negative correlation between miR-224 and ANGPTL1 expression at RNA level (Fig. 5E right graph, Spearman $\mathrm{r}=-0.1585, P=0.0467)$. 


\section{Rescue expression of ANGPTL1 partially reverses the promotion for malignant phenotype mediated by miR-224}

To further determine the role of ANGPTL1 in the promotion effect on malignant phenotype mediated by miR-224, we reintroduced ANGPTL1 expression in miR-224transfected H1299 cells (Fig. 6A, $P<0.001$ ). As shown in Fig. 6B-D, rescue expression of ANGPTL1 partially abolished the promotive effect of miR-224 on cell viability (Fig. 6B, $P<0.001$ ), colony formation (Fig. 6C, $P<0.05$ ), migration and invasion ability (Fig. $6 \mathrm{D}, P<0.05)$. These results indicated that ANGPTL1 rescue reverses the promotive effect of miR-224 on malignant phenotype of NSCLC cells partially.

\section{Discussion}

Lymph node status is of great importance in NSCLC, not only for prognosis but also to guide postoperative therapeutic strategy [16]. Previous reports showed that some miRNAs as molecular markers were correlated with either lymph node metastasis or distance metastasis. MiRNA-130a [17] and miR-196a [18] expression were positively associated with lymph node metastasis, while miRNA-451 [19] expression was negatively associated with lymph node metastasis. MiR-328 [20] was validated to have predictive value for brain metastasis with a sensitivity and specificity of $75 \%$ and $81.8 \%$, respectively. These literature revealed that miRNAs might be correlated with and used to predict lymphatic metastasis of NSCLC. In the present study, we aimed to evaluate miR-224 role on lymph node metastasis of NSCLC. Previously, miRNAs microarray analysis had identified the differentially expressed miRNAs between lymphatic metastatic nodes and noncancerous lymph node from five NSCLC patients [15]. One miRNAs, miR-224, exerted a higher expression in tissues with lymph node metastasis. Here, a validation cohort consisting of 155 NSCLC patients was recruited to validate 
the positive correlation of miR-224 and lymph node metastasis.

MiR-224 had been revealed the involvement of malignant characters regulation in human cancers. It was reported to promote tumor growth and metastasis in gastric [21], colorectal [22], and papillary thyroid cancer [23].In addition, miR-224 was reported to be associated with aggressive progression and poor prognosis in human cervical[24] and colorectal cancer [25]. In colonic cancer, miR-224 was identified as one of miRNAs related to lymph node metastasis [26]. As for lung cancer, in 2014, Zhu et.al. reported that decreased miR-224 expression was found in the cancer tissues and tissues with lymph node metastasis by q-PCR analysis 115 cases of patients, and over-expression of miR-224 in A549 cells by transfected with mimics resulted in a suppression of cell migration and invasion by traswell and wound healing assay[27]. However, contrary to Zhu et.al., in 2015 Cui et.al. published their results in the PNAS that miR-224 was significantly up-regulated in NSCLC tissues, particularly in resected NSCLC metastasis by both q-PCR and in situ hybridization analysis, and increased miR-224 expression promotes cell migration, invasion, and proliferation [28]. In 2020, miR-224 was predicted as one of miRNAs associated with lymph node metastasis for lung adenocarcinoma only by bioinformatics analysis the TCGA and GEO datasets [29]. Here, consistent with the report form Cui et.al, our microarray also identified the miR224 as an upregulation miRNA in the tissues with lymph node metastasis. More important, we also validated its high expression about 7-fold change in tissues with metastasis compared to non-metastasis in our own cohort consisting of 155 cases of patients. Our results from gain-function and loss-function experiments also validated that miR-224 functioned as oncogene in lung cancer, which could promotes cell migration, invasion, and proliferation both in vitro and in vivo.

Previously, some direct targets of miR-224 had been found to be involved in its function, 
such as by directly targeting the tumor suppressors TNF $\alpha$-induced protein 1 (TNFAIP1) and SMAD4 in lung cancer [28]. Here, we reported another direct target, ANGPTL1, and an indirect YPEL1 as downstream regulation genes of miR-224. Previously, ANGPTL1 was reported as a tumor suppressor in lung cancer, which could inhibit cancer cell motility and metastasis by abrogating the expression of the EMT mediator SLUG [30]. Our rescue ANGPTL1 experiments also validated its suppression role in regulation of malignant phenotype in lung cancer, including cell motility. YPEL1 had been identified as a significant down-regulated gene in pancreatic cancer and its reduced expression might be related to perineural invasion and prognosis [31]. However, the underlying mechanism of miR-224 on the regulation of YPEL1 and its role in lung cancer needed more experiments to further investigate.

\section{Conclusions}

Our findings highlight that miR-224 serve as an oncogene associated with lymph node metastasis in NSCLC patients. The promotion effect of miR-224 on the malignant phenotype might be due to its regulation on two tumor suppressors, ANGPTL1 and YPEL1. Thus, miR-224 may provide a predictor for lymph node metastasis of NSCLC.

\section{Additional file}

Additional file: Supplementary Table S1, Supplementary Figure S1.

\section{Abbreviations}

TCGA: The Cancer Genome Atlas; NSCLC: non-small-cell lung cancer; LNM:

Lymph node metastasis; CAM: chick embryo chorioallantoic membrane assay; DEGs: differential expressed genes; qRT-PCR: Real-Time Quantitative Reverse Transcription PCR; CCK-8: Cell Counting Kit-8; ANGPTL: Angiopoietin-like Protein 1; YPEL1: yippee-like 1. 


\section{Declarations}

\section{Ethics approval and consent to participate}

All methods and experiments were approved by the Ethics Committee of Peking University Cancer Hospital \& Institute, and the informed consent was obtained from all the participants. All animal experiments were performed in accordance with guidelines and regulation of Animal Ethics Committee of Peking University Cancer Hospital \& Institute. The study was carried out in compliance with the ARRIVE guidelines. All experiments involving humans were performed in accordance with relevant guidelines and regulations.

\section{Consent to publish}

Not applicable.

\section{Availability of data and materials}

All relevant materials and data are available from corresponding author (Dr. Jinfeng Chen) for non-commercial purposes.

\section{Competing interests}

All authors declare no competing interest.

\section{Funding}

This study was supported by the National Natural Science Foundation of China (Grant No. 81773144,81772632 ), Special funds of the Ministry of Finance for Reform and Development and Science Foundation of Peking University Cancer Hospital 2020-9.

\section{Authors' Contributions}

JC designed the research. HH performed most of the experiments and wrote the manuscript. BP, FL, LW, XL and YY helped the collection and analysis of the clinical data. All authors approved the final manuscript.

\section{Acknowledgements}

Not applicable.

\section{References}


1. Siegel RL, Miller KD, Jemal A: Cancer statistics, 2019. CA: a cancer journal for clinicians 2019, 69(1):7-34.

2. Hirsch FR, Scagliotti GV, Mulshine JL, Kwon R, Curran WJ, Jr., Wu YL, PazAres L: Lung cancer: current therapies and new targeted treatments. Lancet 2017, 389(10066):299-311.

3. Gelatti ACZ, Drilon A, Santini FC: Optimizing the sequencing of tyrosine kinase inhibitors (TKIs) in epidermal growth factor receptor (EGFR) mutation-positive non-small cell lung cancer (NSCLC). Lung cancer 2019, 137:113-122.

4. Yang X, Pan X, Liu H, Gao D, He J, Liang W, Guan Y: A new approach to predict lymph node metastasis in solid lung adenocarcinoma: a radiomics nomogram. Journal of thoracic disease 2018, 10(Suppl 7):S807-S819.

5. Shen-Tu Y, Mao F, Pan Y, Wang W, Zhang L, Zhang H, Cheng B, Guo H, Wang $Z$ : Lymph node dissection and survival in patients with early stage nonsmall cell lung cancer: A 10-year cohort study. Medicine 2017, 96(43):e8356.

6. Riffo-Campos AL, Riquelme I, Brebi-Mieville P: Tools for Sequence-Based miRNA Target Prediction: What to Choose? International journal of molecular sciences 2016, 17(12).

7. Rupaimoole R, Slack FJ: MicroRNA therapeutics: towards a new era for the management of cancer and other diseases. Nature reviews Drug discovery 2017, 16(3):203-222.

8. Fazi F, Fontemaggi G: MicroRNAs and lymph node metastatic disease in lung cancer. Thoracic surgery clinics 2012, 22(2):167-175.

9. Min L, Wang F, Hu S, Chen Y, Yang J, Liang S, Xu X: Aberrant microRNA137 promoter methylation is associated with lymph node metastasis and poor clinical outcomes in non-small cell lung cancer. Oncology letters 2018, 15(5):7744-7750.

10. Chen YJ, Guo YN, Shi K, Huang HM, Huang SP, Xu WQ, Li ZY, Wei KL, Gan TQ, Chen G: Down-regulation of microRNA-144-3p and its clinical value in non-small cell lung cancer: a comprehensive analysis based on microarray, miRNA-sequencing, and quantitative real-time PCR data. Respiratory research 2019, 20 (1):48.

11. Chen Y, Min L, Ren C, Xu X, Yang J, Sun X, Wang T, Wang F, Sun C, Zhang $X$ : miRNA-148a serves as a prognostic factor and suppresses migration and invasion through Wnt1 in non-small cell lung cancer. PloS one 2017, 12(2):e0171751.

12. Cheng XK, Lin WR, Jiang H, Su ZH, Li L, Wang J: MicroRNA-129-5p inhibits invasiveness and metastasis of lung cancer cells and tumor angiogenesis via targeting VEGF. European review for medical and pharmacological sciences 2019, 23(7):2827-2837.

13. Shi R, Chiang VL: Facile means for quantifying microRNA expression by real-time PCR. BioTechniques 2005, 39(4):519-525.

14. Xiao F, Zuo Z, Cai G, Kang S, Gao X, Li T: miRecords: an integrated resource for microRNA-target interactions. Nucleic acids research 2009, 
37(Database issue):D105-110.

15. Wu L, Hu B, Zhao B, Liu Y, Yang Y, Zhang L, Chen J: Circulating microRNA422a is associated with lymphatic metastasis in lung cancer. Oncotarget 2017, 8(26):42173-42188.

16. Lardinois D, De Leyn P, Van Schil P, Porta RR, Waller D, Passlick B, Zielinski M, Lerut T, Weder $\mathrm{W}$ : ESTS guidelines for intraoperative lymph node staging in non-small cell lung cancer. European journal of cardio-thoracic surgery : official journal of the European Association for Cardio-thoracic Surgery 2006, 30(5):787-792.

17. Wang XC, Tian LL, Wu HL, Jiang XY, Du LQ, Zhang H, Wang YY, Wu HY, Li DG, She $Y$ et al: Expression of miRNA-130a in nonsmall cell lung cancer. The American journal of the medical sciences 2010, 340(5):385-388.

18. Liu XH, Lu KH, Wang KM, Sun M, Zhang EB, Yang JS, Yin DD, Liu ZL, Zhou J, Liu ZJ et al: MicroRNA-196a promotes non-small cell lung cancer cell proliferation and invasion through targeting HOXA5. BMC cancer 2012, 12:348.

19. Wang XC, Tian LL, Jiang XY, Wang YY, Li DG, She Y, Chang JH, Meng AM: The expression and function of miRNA-451 in non-small cell lung cancer. Cancer letters 2011, 311(2):203-209.

20. Arora S, Ranade AR, Tran NL, Nasser S, Sridhar S, Korn RL, Ross JT, Dhruv $\mathrm{H}$, Foss KM, Sibenaller Z et al: MicroRNA-328 is associated with (non-small) cell lung cancer (NSCLC) brain metastasis and mediates NSCLC migration. International journal of cancer 2011, 129(11):2621-2631.

21. He C, Wang L, Zhang J, Xu H: Hypoxia-inducible microRNA-224 promotes the cell growth, migration and invasion by directly targeting RASSF8 in gastric cancer. Molecular cancer 2017, 16(1):35.

22. Yuan K, Xie K, Fox J, Zeng H, Gao H, Huang C, Wu M: Decreased levels of miR-224 and the passenger strand of miR-221 increase MBD2, suppressing maspin and promoting colorectal tumor growth and metastasis in mice. Gastroenterology 2013, 145(4):853-864 e859.

23. Zang CS, Huang HT, Qiu J, Sun J, Ge RF, Jiang LW: MiR-224-5p targets EGR2 to promote the development of papillary thyroid carcinoma. European review for medical and pharmacological sciences 2020, 24(9):48904900.

24. Shen SN, Wang LF, Jia YF, Hao YQ, Zhang L, Wang H: Upregulation of microRNA-224 is associated with aggressive progression and poor prognosis in human cervical cancer. Diagnostic pathology 2013, 8:69.

25. Adamopoulos PG, Kontos CK, Rapti SM, Papadopoulos IN, Scorilas A: miR224 overexpression is a strong and independent prognosticator of shortterm relapse and poor overall survival in colorectal adenocarcinoma. International journal of oncology 2015, 46(2):849-859.

26. Wang YX, Zhang XY, Zhang BF, Yang CQ, Chen XM, Gao HJ: Initial study of microRNA expression profiles of colonic cancer without lymph node metastasis. Journal of digestive diseases 2010, 11(1):50-54. 
27. Zhu D, Chen H, Yang X, Chen W, Wang L, Xu J, Yu L: Decreased microRNA224 and its clinical significance in non-small cell lung cancer patients. Diagnostic pathology 2014, 9:198.

28. Cui R, Meng W, Sun HL, Kim T, Ye Z, Fassan M, Jeon YJ, Li B, Vicentini C, Peng Y et al: MicroRNA-224 promotes tumor progression in nonsmall cell lung cancer. Proceedings of the National Academy of Sciences of the United States of America 2015, 112(31):E4288-4297.

29. Wang Y, Shang S, Yu K, Sun H, Ma W, Zhao W: miR-224, miR-147b and miR31 associated with lymph node metastasis and prognosis for lung adenocarcinoma by regulating PRPF4B, WDR82 or NR3C2. PeerJ 2020, 8:e9704.

30. Kuo TC, Tan CT, Chang YW, Hong CC, Lee WJ, Chen MW, Jeng YM, Chiou J, Yu P, Chen PS et al: Angiopoietin-like protein 1 suppresses SLUG to inhibit cancer cell motility. The Journal of clinical investigation 2013, 123(3):1082-1095.

31. Abiatari I, Kiladze M, Kerkadze V, Friess H, Kleeff J: Expression of YPEL1 in pancreatic cancer cell lines and tissues. Georgian medical news 2009(175):60-62. 


\section{Figure legends}

Fig. 1. MiR-224 was highly expressed in NSCLC tissues with lymphatic metastasis. (A) Heat map for miR-224 expression in lymphatic metastasis positive and negative tissues by microarray analysis. (B) MiR-224 expression in lymphatic metastasis positive and negative tissues by q-PCR analysis. (C) ROC curve analysis for prediction of lymphatic metastasis by miR-224 expression. (D) Survival analysis by the Kaplan-Meier method according to miR-224 expression.

Fig. 2. Over-expression of miR-224 enhanced cell viability, colony formation, migration and invasion in H1299 cells in vitro. (A,B) miR-224 expression in lung cancer cells were detected by q-PCR. (C) Cell viability for miR-224 overexpression and NC control cells were determined by CCK8 agent. (D) Plate colony formation for miR-224 overexpression and NC control cells. (E) Cell spreading ability calculated as wound closure of monolayer were determined by wound healing assay for different cells. (F)Cell migration and invasion ability quantified by cell numbers were accessed by Transwell assay without or with Matrigel, Scale bars, $200 \mu \mathrm{m}$. The data in B, D, E, F were presented as means $\pm \mathrm{SD}$ and statistical significance were shown as $* * P<0.01$, $* * * P<0.001$.

Fig. 4. Inhibition of miR-224 suppresses PC9 cell migration and invasion in vitro. (A) miR-224 expression in deferent cells were detected by q-PCR. (B) CCK-8 assays were performed to access cell viability (C) Plate colony formation assays were performed to monitor cell growth. (E) Wound healing assay to study cell spreading ability and wound closure of monolayer were calculated. (F) Transwell assays were performed to determine cell migration and invasion. The number of cells was counted in four 
different fields. Scale bars, $100 \mu \mathrm{m}$. The data in C, D, E were presented as means \pm SD and statistical significance were shown as ** $P<0.01$, *** $P<0.001$.

Fig.5. MiR-224 functions as oncomiR in vivo. A, C. Control cells or cells with mimics or inhibitors were inoculated onto the CAM. The effect of miR-224 on tumor growth was evaluated based on the tumor weight of each group. B, D. Representative pictures of lung metastasis lesion by a laser scanning confocal microscope. The effect of miR224 on lung metastasis was evaluated by the number of metastatic lesion (tumor cells were labeled with red fluorescent dye CM-Dil ). The data in the bar graphs were calculated as the mean \pm S.D. for each group.

Fig.6. ANGPTL1 is a direct target of miR-224. (A)Venn diagram predicted the candidate targets of miR-224. (B) Western blot analysis of the ANGPTL1 and YPEL1 expression in miR-224 mimics or inhibitors cells. C. Schematic model indicates complementary binding sites and corresponding mutation of miR-224 for ANGPTL1 and YPEL1 3'UTR. D. Wild or mutant type PGL3-control plasmids were transfected into HEK293T cells together with miR-224 mimic or NC. Firefly fluorescence intensity (FL) normalized by Renilla fluorescence (RL) intensity was calculated after $24 \mathrm{~h}$ transfection. E. Spearman correlation analysis of the miR-224 and ANGPTL1 expression in TCGA-LUAD database and in our cohort of lung cancer consisting of 155 cases. The data in $\mathrm{D}$ are presented as means $\pm \mathrm{SD}$ and statistical significance were shown as $* * P<0.01$, ns: not significance.

Fig. 6. Figure 5. ANGPTL1 rescue expression partially diminishes the miR-224mediated promotion effect on malignant phenotype of H1299 cell. A.Western blot 
determines ANGPTL1 rescue expressing. B. CCK-8 assays were performed to determine cell viability. C. Plate colony formation assays were performed to detect cell growth. D. Transwell assays were used to monitor cell migration and invasion.

The number of cells was counted in four different fields. Scale bars, $200 \mu \mathrm{m}$. The data in $\mathrm{C}, \mathrm{D}$ were presented as means $\pm \mathrm{SD}$ and statistical significance were shown as $* P<0.05,{ }^{* *} P<0.01, * * * P<0.001$. 


\section{Figures}
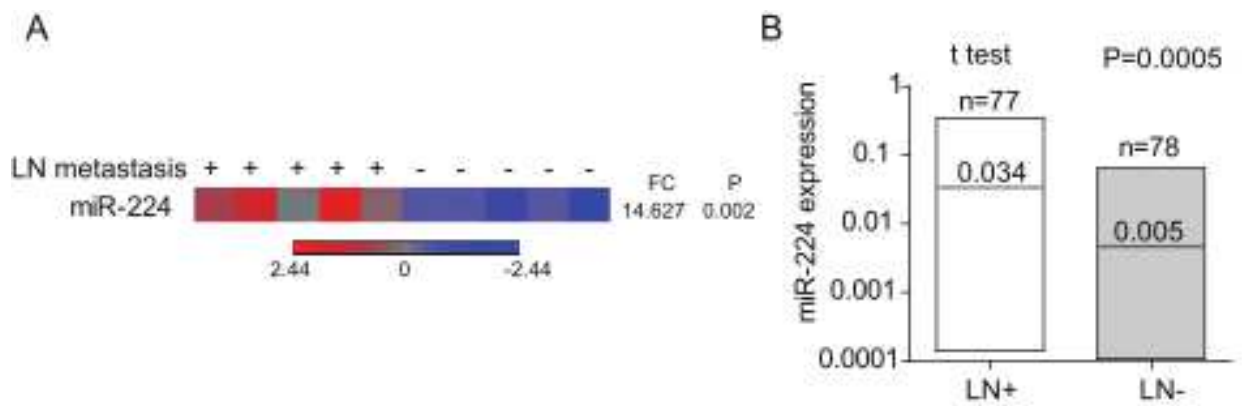

C

D
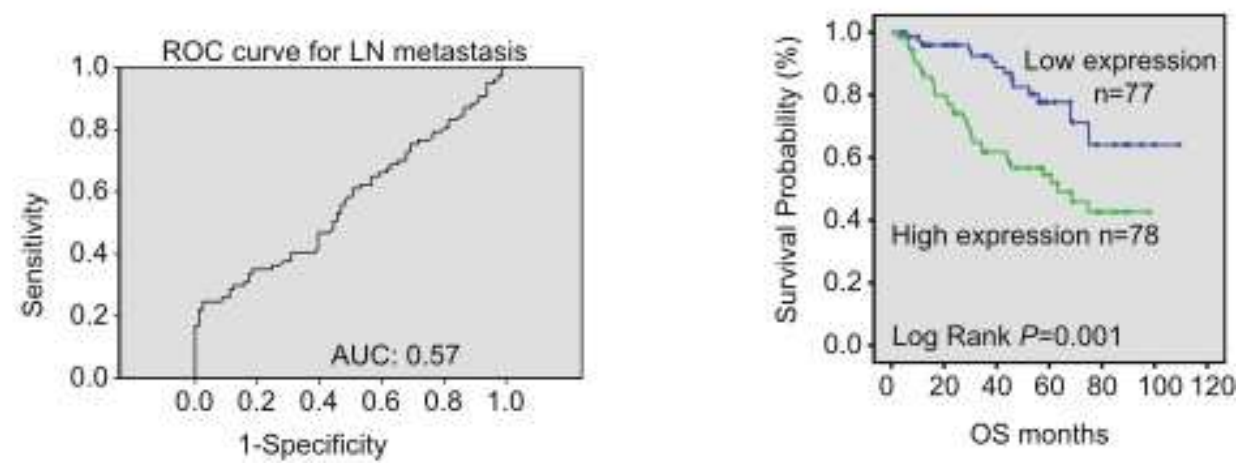

\section{Figure 1}

MiR-224 was highly expressed in NSCLC tissues with lymphatic metastasis. (A) Heat map for miR-224 expression in lymphatic metastasis positive and negative tissues by microarray analysis. (B) MiR-224 expression in lymphatic metastasis positive and negative tissues by q-PCR analysis. (C) ROC curve analysis for prediction of lymphatic metastasis by miR-224 expression. (D) Survival analysis by the Kaplan-Meier method according to miR-224 expression. 
A

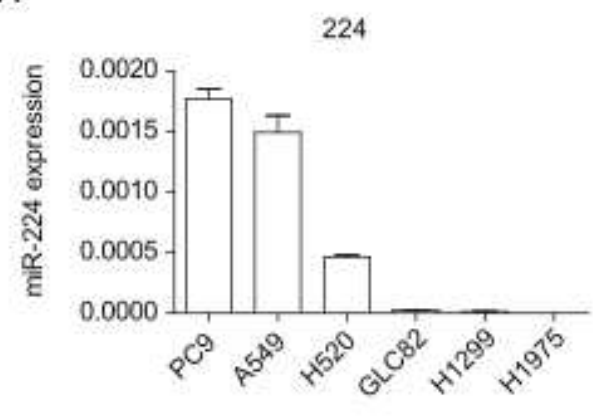

224

B

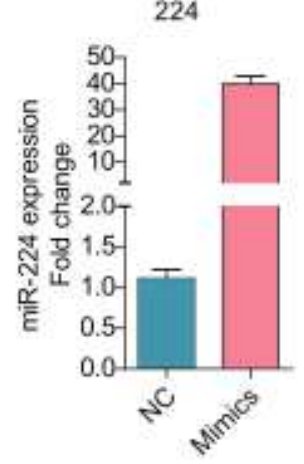

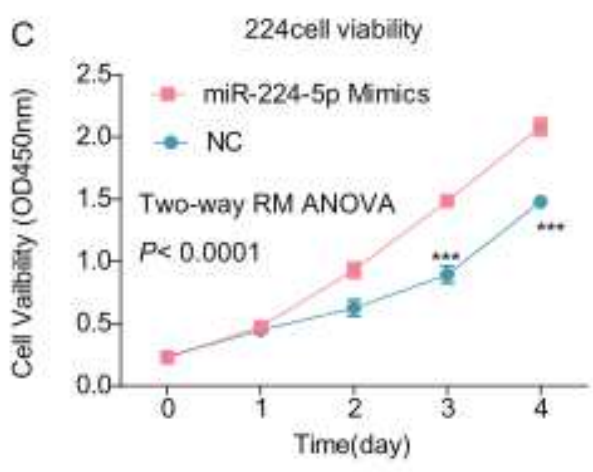

D
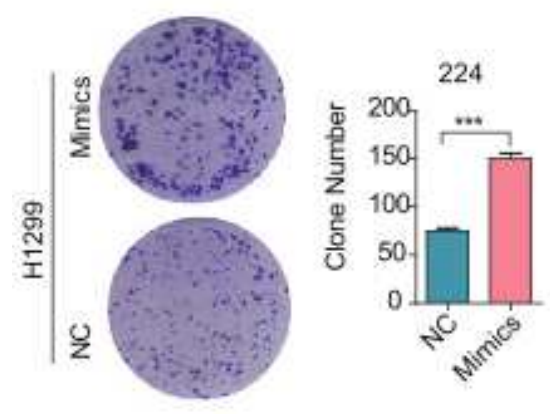

$E$

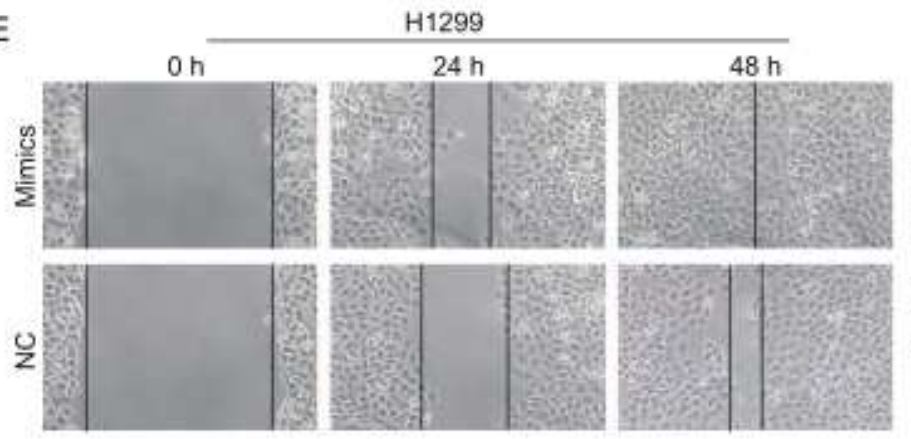

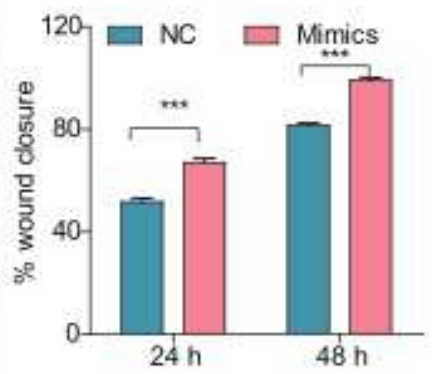

$\mathrm{F}$
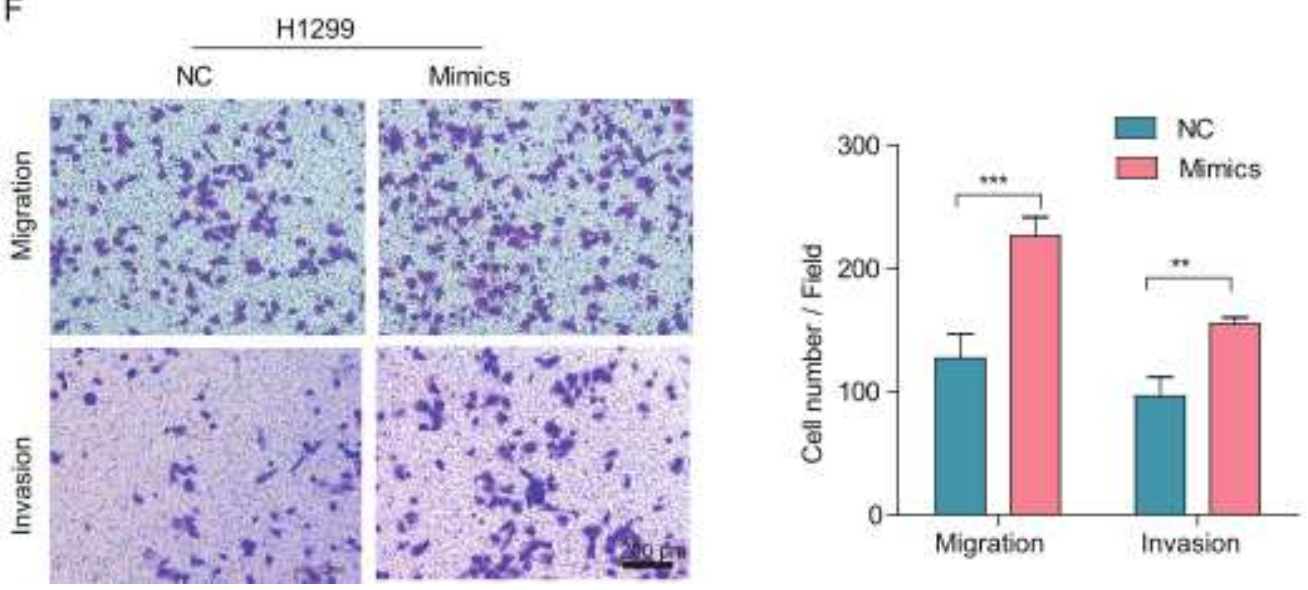

Figure 2

Over-expression of miR-224 enhanced cell viability, colony formation, migration and invasion in $\mathrm{H} 1299$ cells in vitro. $(A, B)$ miR-224 expression in lung cancer cells were detected by q-PCR. (C) Cell viability for miR-224 overexpression and NC control cells were determined by CCK8 agent. (D) Plate colony formation for miR-224 overexpression and NC control cells. (E) Cell spreading ability calculated as wound closure of monolayer were determined by wound healing assay for different cells. (F)Cell migration and invasion ability quantified by cell numbers were accessed by Transwell assay without or with Matrigel, Scale bars, $200 \mu \mathrm{m}$. The data in $B, D, E, F$ were presented as means \pm SD and statistical significance were shown as $\star \star P<0.01, * \star * P<0.001$. 


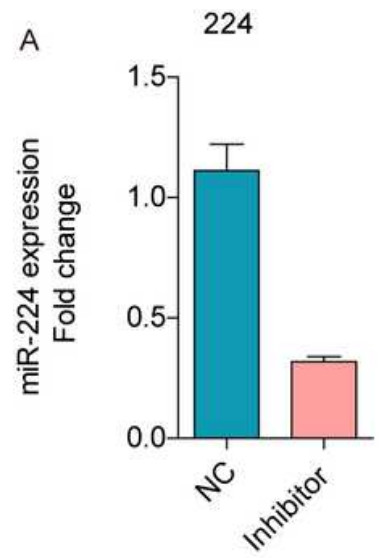

B

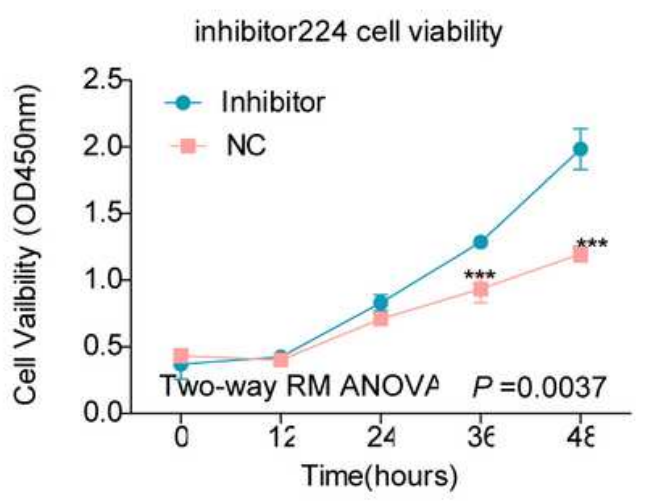

C

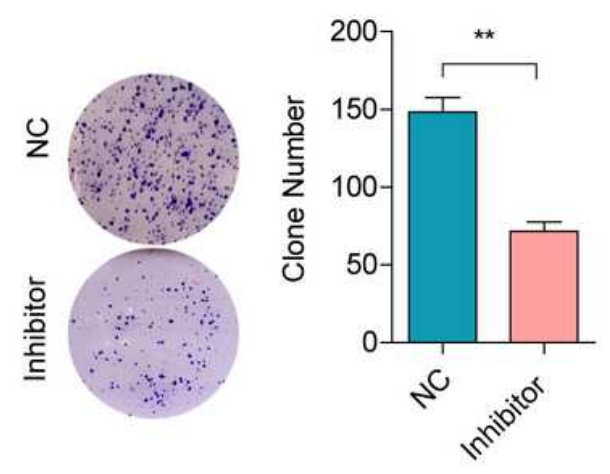

D
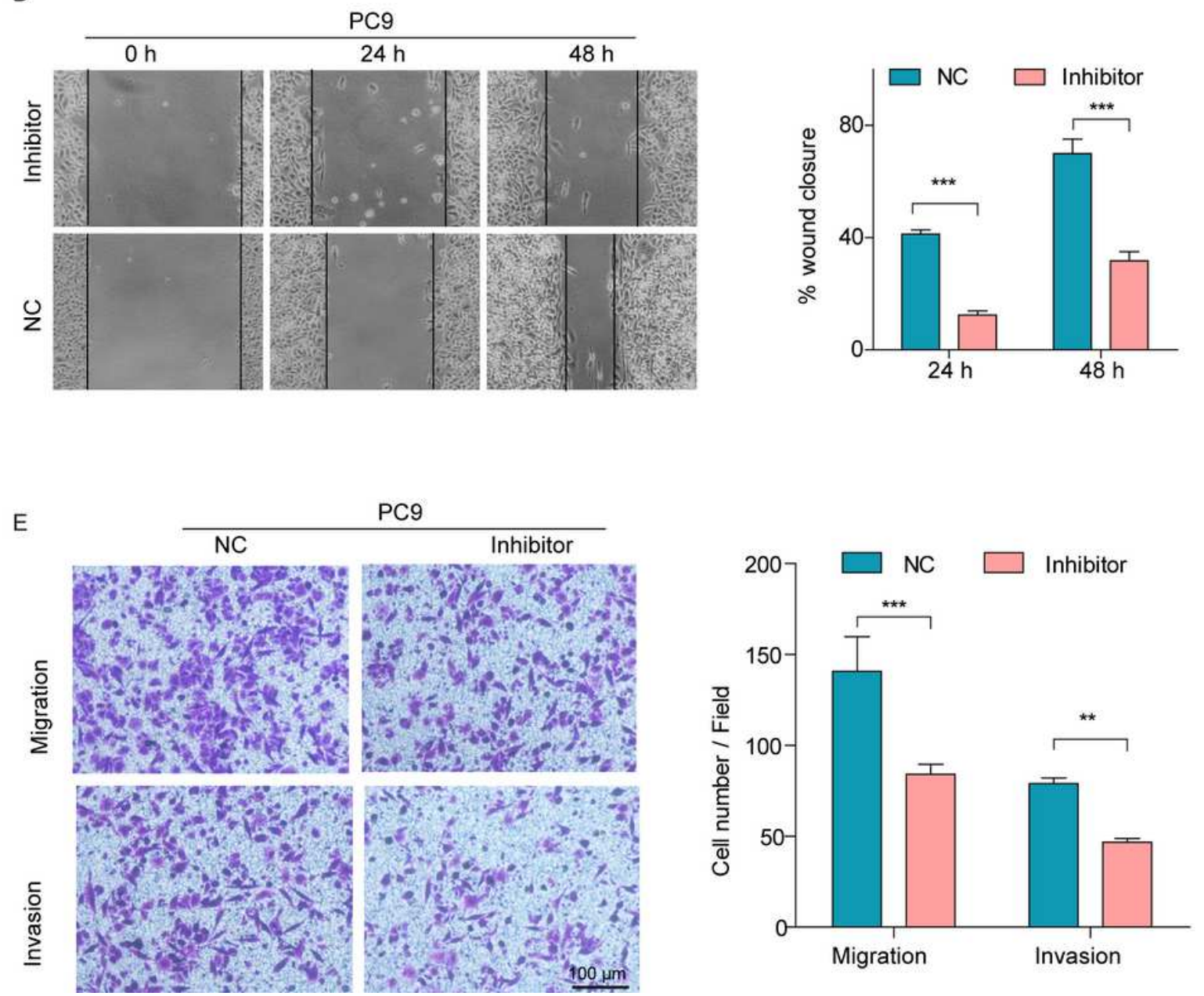

Figure 3

Inhibition of miR-224 suppresses PC9 cell migration and invasion in vitro. (A) miR-224 expression in deferent cells were detected by q-PCR. (B) CCK-8 assays were performed to access cell viability (C) Plate colony formation assays were performed to monitor cell growth. $(E)$ Wound healing assay to study cell spreading ability and wound closure of monolayer were calculated. (F) Transwell assays were performed to determine cell migration and invasion. The number of cells was counted in four different fields. Scale 
bars, $100 \mu \mathrm{m}$. The data in $C, D, E$ were presented as means \pm SD and statistical significance were shown as ** $P<0.01, * * * P<0.001$.

A

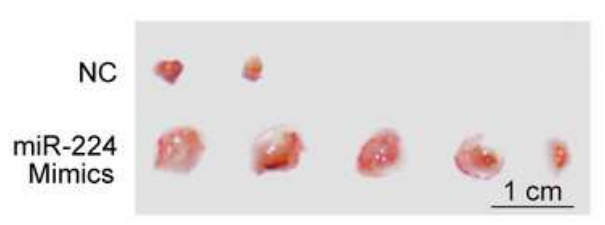

C

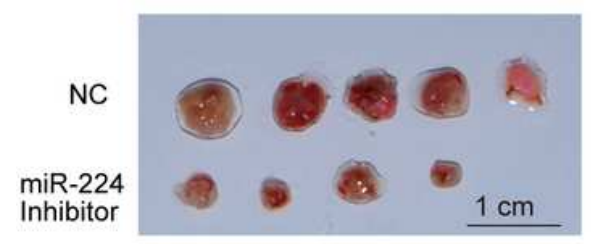

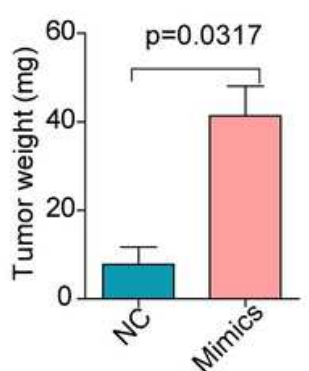

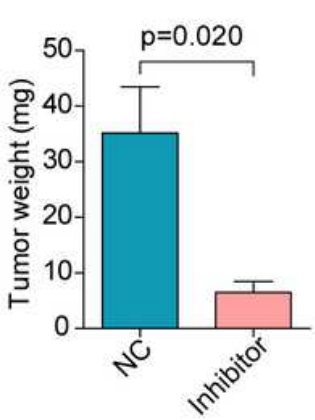

B
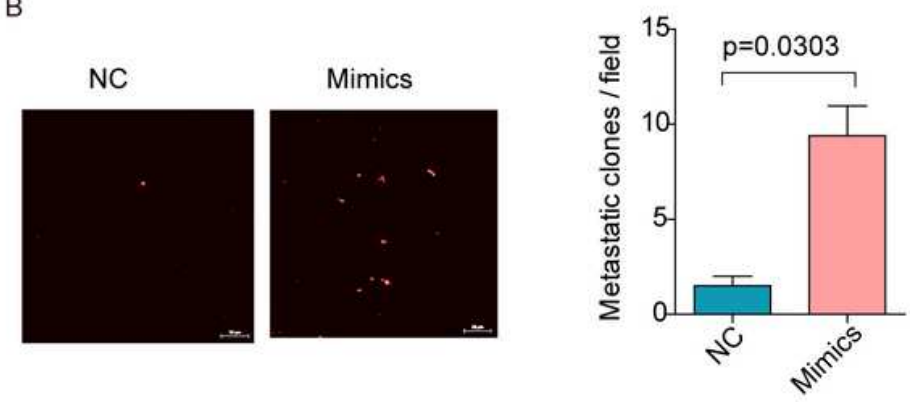

D

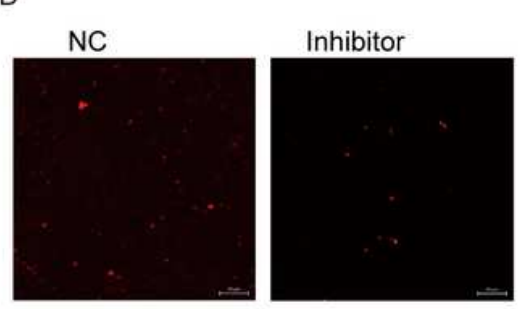

Figure 4

MiR-224 functions as oncomiR in vivo. A, C. Control cells or cells with mimics or inhibitors were inoculated onto the CAM. The effect of miR-224 on tumor growth was evaluated based on the tumor weight of each group. B, D. Representative pictures of lung metastasis lesion by a laser scanning confocal microscope. The effect of miR-224 on lung metastasis was evaluated by the number of metastatic lesion (tumor cells were labeled with red fluorescent dye CM-Dil). The data in the bar graphs were calculated as the mean \pm S.D. for each group. 
A

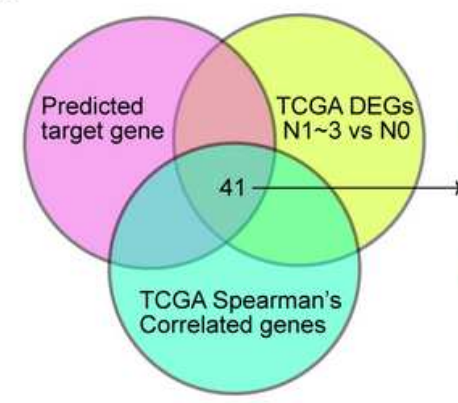

C

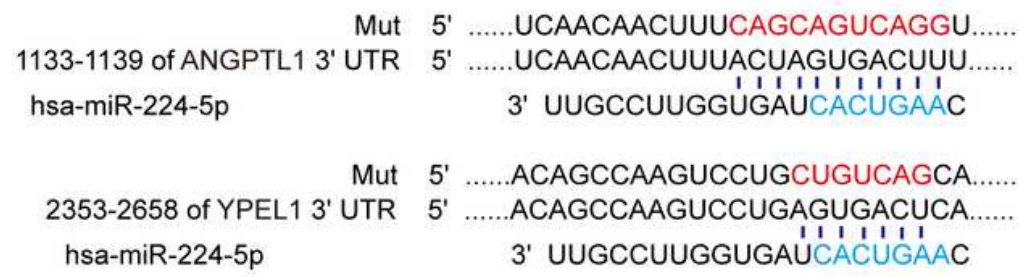

FNBP1L; AOC3; FZD4; FHL5; ATOH8; ZNF667; TFB1M;MEGF11; MOXD1; DKK2: ZNF 43: C10orf46; MEX3A: YPEL1; PIK3R3; CAB39L; RBM3; ASB5; SF3A3: CD69; METTL10; ZNF781; SLC4A4; D3; DUSP5; MLPH; VAV3; ZNF91: PDCD2; PCMT1; RGS13;SNX22; SLC26A9
B
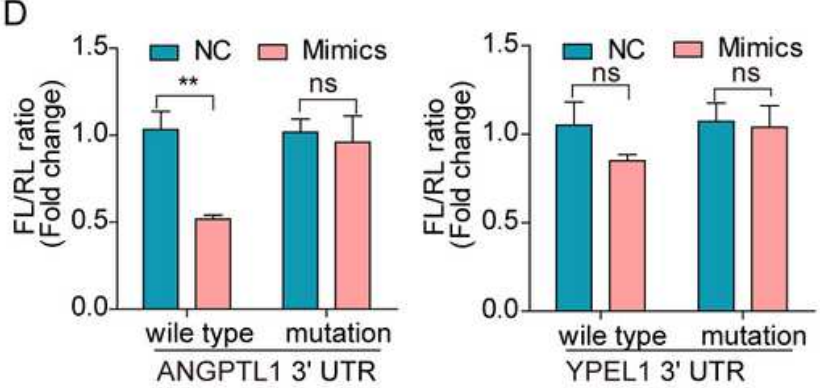
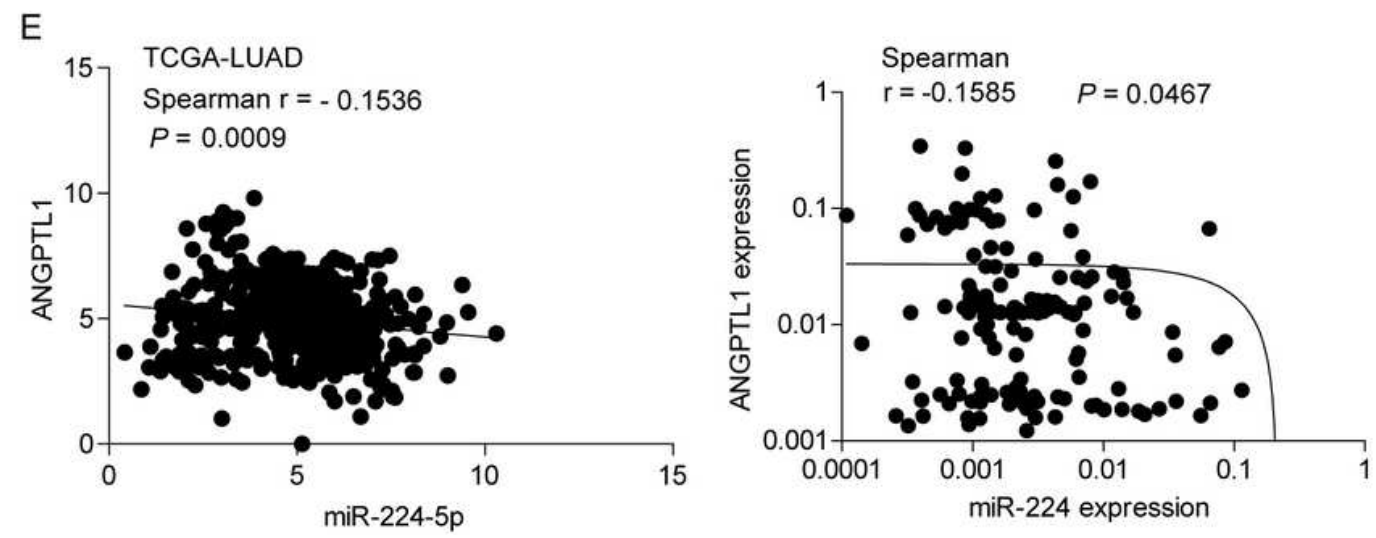

\section{Figure 5}

ANGPTL1 is a direct target of miR-224. (A)Venn diagram predicted the candidate targets of miR-224. (B) Western blot analysis of the ANGPTL1 and YPEL1 expression in miR-224 mimics or inhibitors cells. C. Schematic model indicates complementary binding sites and corresponding mutation of miR-224 for ANGPTL1 and YPEL1 3'UTR. D. Wild or mutant type PGL3-control plasmids were transfected into HEK293T cells together with miR-224 mimic or NC. Firefly fluorescence intensity (FL) normalized by Renilla fluorescence $(\mathrm{RL})$ intensity was calculated after $24 \mathrm{~h}$ transfection. E. Spearman correlation analysis of the miR-224 and ANGPTL1 expression in TCGA-LUAD database and in our cohort of lung cancer consisting of 155 cases. The data in D are presented as means \pm SD and statistical significance were shown as ** $P<0.01$, ns: not significance. 

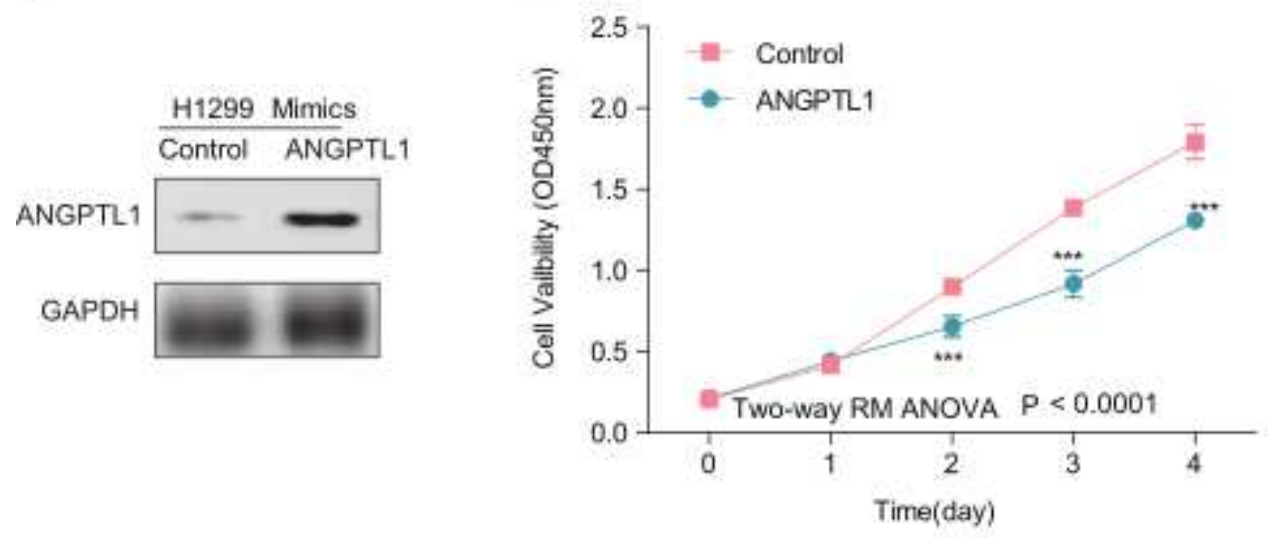

C
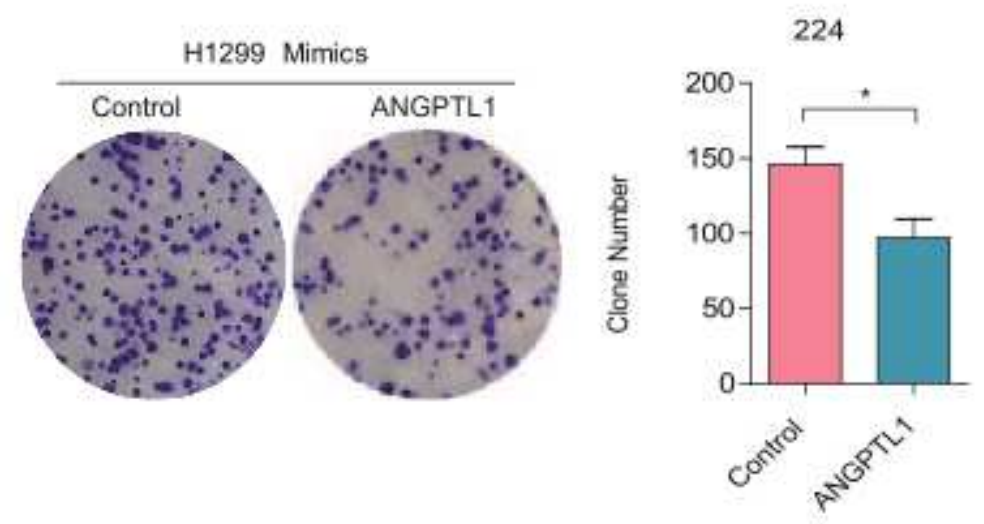

D
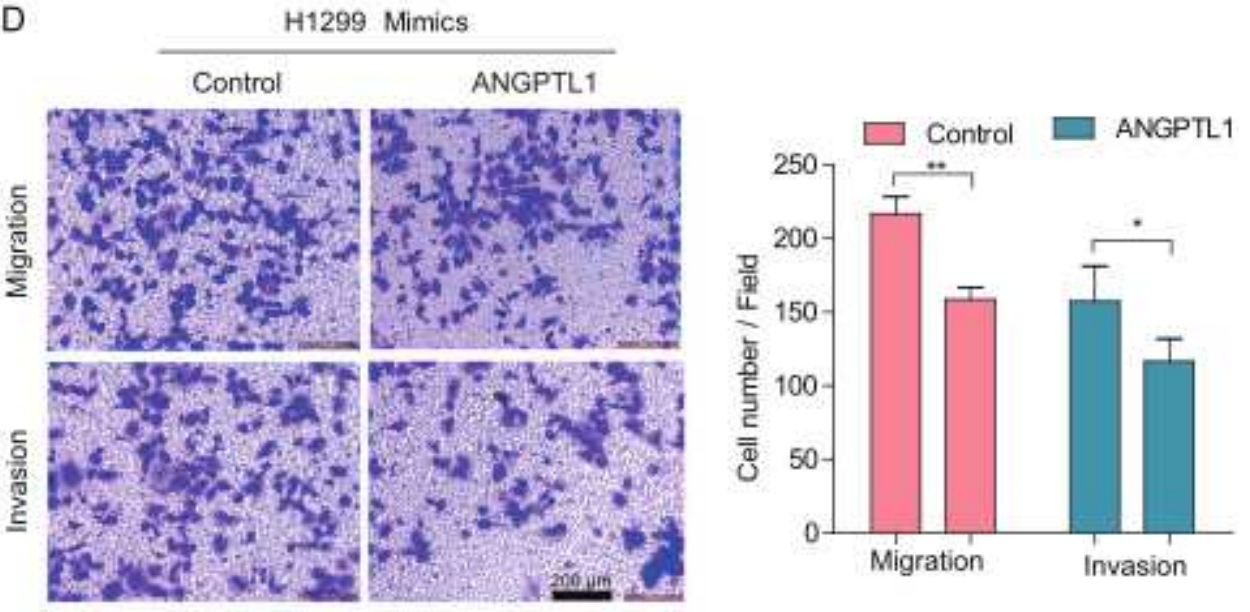

Figure 6

ANGPTL1 rescue expression partially diminishes the miR-224-mediated promotion effect on malignant phenotype of $\mathrm{H} 1299$ cell. A.Western blot determines ANGPTL1 rescue expressing. B. CCK-8 assays were performed to determine cell viability. C. Plate colony formation assays were performed to detect cell growth. D. Transwell assays were used to monitor cell migration and invasion. The number of cells was counted in four different fields. Scale bars, $200 \mu \mathrm{m}$. The data in C,D were presented as means $\pm S D$ and statistical significance were shown as $* P<0.05,{ }^{\star \star} P<0.01, * \star \star P<0.001$.

\section{Supplementary Files}


This is a list of supplementary files associated with this preprint. Click to download.

- SupplementaryTableS1primersequence.docx

- originalwb.docx 